\title{
Oscillation regimes of gas/vapor bubbles
}

\author{
L. Bergamasco ${ }^{\mathrm{a}, \mathrm{b}}$, D. Fuster ${ }^{\mathrm{a}, \mathrm{b}}$ \\ ${ }^{a}$ Sorbonne Universités, UPMC Univ Paris 06, UMR 7190, \\ Institut Jean Le Rond d'Alembert, F-75005, Paris, France \\ ${ }^{b}$ CNRS, UMR 7190, Institut Jean Le Rond d'Alembert, F-75005, Paris, France
}

\begin{abstract}
In this work we investigate the effect of heat and mass transfer on the dynamics of gas-vapor bubbles. We present phase diagrams for the bubble oscillation regimes, which are built by comparison of various models with different level of simplification for an air-water system. These diagrams show the range of validity of the simplifying assumptions on the Peclet-number/vapor-content plane, providing an insight on the physical process which regulates the bubble response with respect to external pressure perturbations. The analysis is presented for both the linear and weakly nonlinear regime. In the former case we use linearized solutions of the full system; in the latter, numerical simulations validated against the analytical solutions in the linear limit. We show that even at very low frequencies, there exist regimes where transient diffusion effects arise and restrict the applicability of the commonly-adopted assumption of full-equilibrium conditions inside the bubble. Non-linearity is found to restrict even further the range of applicability of this hypothesis, due to the variation of the vapor content beyond a critical value.
\end{abstract}

Keywords: Phase change, Bubble oscillation, Evaporation

\section{INTRODUCTION}

The response of liquids containing bubbles to external pressure changes has important implications in engineering, geophysical and biomedical applications [1,2]. Dilute systems in which bubbles contain a negligible amount of vapor have been extensively investigated, both theoretically and experimentally (see [3] for a review). However, the consequences of phase transition in liquids containing bubbles with an appreciable amount of vapor are not completely understood. Although theoretical and experimental studies show that heat- and mass-transfer effects have a non-negligible influence on the bubble response [4] and thus, on the overall fluid properties [5, 6, 7], it is also possible to find conditions where mass-transfer effects are not evident and/or more difficult to capture [8]. Available works in the literature propose different quantities to determine whether mass-transfer effects are relevant or not $[9,10]$ but the problem is that a solid base for modeling still lacks [11]. Systematic approaches for dilute mixtures with bubbles containing vapor in addition to a permanent gas have been proposed only recently $[11,12,13]$. In these latter works, the authors firstly address the response of a single bubble to a varying pressure field considering heatand mass-transfer effects, and then discuss the implica- tions on the speed of sound in the mixture.

The development of numerical codes able to correctly predict the response of bubbles undergoing phase change is challenging. One possibility for fast bubble oscillations is to assume that the influence of both the heat and the mass flux across the interface on the bubble's pressure are negligible compared to the pressure changes imposed by the gas volume change. In this case the bubble response is adiabatic and one can relate volume and pressure changes through a polytropic transformation. At the other extreme, for very slow pressure/temperature variations, it can be assumed that the bubble reaches a thermodynamic equilibrium with its surroundings so that the vapor pressure is uniform and solely given by the system's temperature. Within these two limiting solutions, the mass flux across the interface is influenced by the diffusion of mass and heat both in the liquid surrounding the bubble and inside the bubble playing an important role on how the bubble's pressure change as a function of volume. Unfortunately, the definition of the relevant dimensionless parameters that determine the relevance of various mechanisms on the bubble's response and the total mass flux is not straightforward and it is difficult to find in the literature quantitative studies about the range 
of validity of various assumptions. In this view, the spherically symmetric assumption provides a simple yet interesting situation to clarify important phenomena about the dynamic response of a single bubble with respect to external pressure/temperature perturbations. Using this framework, several phenomena have already been investigated using simplified models based on the Rayleigh-Plesset equation and an effective equation of state that relates the bubble's pressure and volume changes. For instance, it is possible to derive analytical expressions for the resonance frequency and damping factor for pure-gas bubbles oscillating in the linear limit (reviewed in [14]); heat-transfer, rectified diffusion and secondary resonance frequency for pure-vapor bubbles $[15,16,17,18,19]$ and the effect of soluble or insoluble gas on the dynamics of vapor bubbles [20, 21, 22]. Fuster \& Montel [12] have recently proposed an analytical derivation of the resonance frequency and damping factor for gas-vapor bubbles.

This manuscript presents an analysis of heat- and mass-transfer effects on the dynamic response of gasvapor bubbles. The manuscript is structured as follows. Firstly, we address the problem of linear oscillations and propose phase diagrams for the bubble oscillation regimes, which are built by the comparison between different simplified models and the full analytical solution for gas-vapor bubbles. These diagrams show the range of applicability of the various simplifying modeling assumptions, providing new insight into the transport phenomena which control the physical response of the bubble with respect to the vapor content and the external pressure perturbation. Firstly, we discuss the regimes on the Peclet-number/vapor-quantity plane for the transfer function (which relates the bubble radius oscillation with the external perturbation) and show that, even for very low frequencies, transient effects can prevent the commonly-adopted assumption of full-equilibrium conditions inside the bubble. Secondly, we explore the regimes beyond the linear limit using numerical solutions. The code, which is validated against the analytical solution in the linear limit, allows us to analyze the response of the bubble for various pressure amplitudes and to show the orbits described by the local quantities on the phase diagrams. Non-linearity is found to restrict the range of applicability of the full-equilibrium assumption when local orbits span into other regimes.

\section{PHYSICAL MODEL}

\subsection{Governing equations}

We consider a spherically-symmetric, non-reacting, gas-vapor bubble standing in a pure liquid. The model relies on the mass, momentum, energy and species conservation equations [23]. Integrating the mass and momentum equations in the liquid yields the well-known Rayleigh-Plesset equation, which, neglecting the compressibility of the liquid while considering mass transfer effects reads as [24]:

$$
\begin{aligned}
R \ddot{R}= & -\frac{3}{2}\left(\dot{R}-\frac{J}{\rho_{l}}\right)^{2}+\frac{R \dot{J}}{\rho_{l}}+2 \frac{J}{\rho_{l}}\left(\dot{R}-\frac{J}{\rho_{l}}\right) \\
& +\frac{p_{b}-p_{\infty}}{\rho_{l}}-\frac{2 \sigma}{\rho_{l} R}-\frac{4 \mu_{l}}{\rho_{l}} \frac{\dot{R}}{R} .
\end{aligned}
$$

In the above equation, $R$ is the radius of the bubble, $J$ the vapor-mass flux across the interface, $\rho_{l}$ the liquid density, $p_{b}$ the bubble pressure (assumed to be uniform), $p_{\infty}$ the far-field liquid pressure, $\sigma$ the surface tension and $\mu_{l}$ the viscosity of the liquid. The internal pressure is assumed to obey the ideal gas law $p_{b}=\rho_{b} \mathcal{R}_{b} T_{b}$, being $\rho_{b}$ the density inside the bubble and $\mathcal{R}_{b}$ the average specific gas constant for the gas/vapor mixture. The energy and species conservation equations in the radial coordinate are:

$$
\begin{aligned}
& \frac{D T}{D t}=\frac{1}{\rho c_{p}} \frac{D p}{D t}+\Gamma_{t} \nabla_{r}^{2} T ; \\
& \rho \frac{D Y}{D t}=\Gamma_{m} \nabla_{r} \cdot\left(\rho \nabla_{r} Y\right) ;
\end{aligned}
$$

where $c_{p}$ is the specific heat, $Y$ the vapor molar fraction, $\Gamma_{t}$ and $\Gamma_{m}$ are the thermal and mass diffusivities respectively and $r$ is the radial coordinate. The properties of the gas/vapor mixture are computed from those of the pure substances using an arithmetic average. The total derivative for a generic (scalar or vector) quantity $\phi$ is defined as $D \phi=\partial_{t} \phi+v_{r} \partial_{r} \phi$, being $v_{r}$ the radial velocity.

The energy equation (2) is solved both inside the bubble and in the surrounding liquid, while the species conservation equation (3) is solved only for the vapor content inside the bubble, as in this work we neglect the gas solubility in the liquid. We remark as this latter approximation implies to neglect the rectified diffusion due to the gas intake inside the bubble [25, 26]; this effect arises in second order and becomes relevant only for very large time scales [27]. In this work we focus on time scales much shorter than those where rectified diffusion effects play a role, which allows us to assume 
this effect to be negligible.

The radial velocity profile inside the bubble is obtained from the continuity equation, which can be rewritten using the energy equation as [3]:

$$
v_{b}(r)=\frac{1}{\gamma p_{b}}\left(\left.(\gamma-1) \lambda_{b} \frac{\partial T_{b}}{\partial r}\right|_{r=R}-\frac{1}{3} \dot{p}_{b} r\right)
$$

with $\gamma$ being the polytropic index (ratio of specific heats) and $\lambda_{b}$ the averaged thermal conductivity of the species inside the bubble.

In order to close the problem, an additional equation is required. One possibility is to impose that the interface of the bubble is in equilibrium with the surrounding liquid at every instant. In this case, the vapor concentration is given by the Clausius-Clapeyron equation. Another possibility is to account for kinetic mass transfer effects using the Hertz-Knudsen-Langmuir (HKL) equation, which imposes the mass transfer flux as a function of the difference between the instantaneous vapor pressure and the equilibrium pressure at the interface's conditions. It can be shown that the model accounting for kinetic mass transfer effects converges to the model assuming equilibrium conditions when either vapor diffusion or heat transfer controls the overall mass transfer rate [12]. In this work we focus on these latter conditions, where the kinetics of the phase change does not have important contribution; however, for practical purposes, we retain the HKL model in the implementation of the equations in the numerical code with an accommodation coefficient equal to 0.35 (see [23] for further details).

\subsection{Boundary conditions}

At the bubble center, the boundary conditions for the energy equation (2) and the species equation (3) are imposed by spherical symmetry as a zero-flux condition:

$$
\left.\frac{\partial T_{b}}{\partial r}\right|_{r=0}=0 \quad \text { and }\left.\quad \frac{\partial Y}{\partial r}\right|_{r=0}=0 .
$$

At the bubble interface, the temperature profile is assumed to be continuous, so $T_{b}(r=R)=T_{l}(r=R)$. The energy balance yields:

$$
\left.\lambda_{l} \frac{\partial T_{l}}{\partial r}\right|_{r=R}=\left.\lambda_{b} \frac{\partial T_{b}}{\partial r}\right|_{r=R}+J H_{v}
$$

with $H_{v}$ being the enthalpy of vaporization/condensation at the interface's temperature. We remark that the mass and momentum conservation at the interface have been directly applied in the derivation of Eq. (1), where the pressure far from the bubble is known. For species, the boundary condition at the interface is given by the continuity of the vapor mass:

$$
\left.\left.\rho_{b}\right|_{r=R} \Gamma_{m, b} \frac{\partial Y}{\partial r}\right|_{r=R}=J\left(1-\left.Y\right|_{r=R}\right) .
$$

Finally, in the far field, the liquid temperature is imposed to be equal to the bulk reference temperature $T_{\infty}$.

\section{NUMERICAL METHOD}

\subsection{Implementation}

The physical model described in Section 2 is numerically solved using the code Bubbles. The code is a Fortran 90 library for the numerical solution of ordinary and partial differential equations, which we developed and tailored for our applications on the basis of a previous code [28]. Spatial integration relies on a stabilized Finite Element Method (FEM) and uses an ArbitraryLagrangian-Eulerian (ALE) framework. The code now disposes of several explicit and implicit time-integration schemes. In this work we adopt an explicit Runge-Kutta method (4-th order) with predictor-corrector algorithms.

\subsection{Validation}

In order to validate the code, we run simulations covering a wide range of forcing frequencies and various vapor contents. The bubble is forced with a sinusoidal pressure wave $p_{\infty}(t)=p_{\infty}\left(1-\Delta \widetilde{p}_{\infty} \sin (\omega t)\right)$, with $\Delta \widetilde{p}_{\infty} \ll 1$. In this situation there exists an analytical solution of the physical model [12] that is summarized next. In the frequency domain, the dimensionless radius oscillation is a function of the external pressure perturbation as:

$$
\Delta \widetilde{R}=-\frac{1}{\widetilde{\omega}_{0}^{2}-\widetilde{\omega}^{2}+2 \widetilde{i \delta \widetilde{\omega}}} \Delta \widetilde{p}_{\infty}
$$

being the variables in the form $y=y_{0}\left(1+\Delta \widetilde{y} \mathrm{e}^{i \omega t}\right)$, with $\Delta \widetilde{y} \ll 1$ for the linear solution to be valid. The parameters are made dimensionless with the characteristic angular frequency $\omega_{c}^{2}=p_{\infty} /\left(\rho_{l} R_{0}^{2}\right)$ so that the resonance angular frequency and damping factor are:

$$
\begin{aligned}
\widetilde{\omega}_{0}^{2} & =\frac{p_{b, 0}}{p_{\infty}} \mathfrak{R}(\Phi)-\frac{2 \sigma}{p_{\infty} R_{0}}+\frac{\rho_{b} \omega^{2} R_{0}^{2}}{p_{\infty}} \mathfrak{I}\left(\widetilde{J}_{0}\right) ; \\
\widetilde{\delta} \widetilde{\omega} & =\frac{p_{b, 0}}{p_{\infty}} \mathfrak{J}(\Phi)+4 \frac{\omega \mu_{l}}{p_{\infty}}-\frac{\rho_{b} \omega^{2} R_{0}^{2}}{p_{\infty}} \mathfrak{R}\left(\widetilde{J}_{0}\right) .
\end{aligned}
$$

In the above parameters, $\Phi$ is the transfer function 
Table 1: Summary of the dimensionless variables used in this work and their definition.

\begin{tabular}{lccc}
\hline NAME & SYMBOL & BUBBLE & LIQUID \\
\hline Peclet number & $\mathrm{Pe}$ & $\omega R_{0}^{2} / \Gamma_{t, b}$ & $\omega R_{0}^{2} / \Gamma_{t, l}$ \\
Sherwood number & $\mathrm{Sh}$ & $\omega R_{0}^{2} / \Gamma_{m, b}$ & - \\
Enthalpy of vaporization & $\widetilde{H}$ & $H_{v} /\left(c_{p, b} T_{\infty}\right)$ & - \\
Conductivity ratio & $\widetilde{\lambda}$ & $\lambda_{b} / \lambda_{l}$ & - \\
Vapor mass fraction & $Y_{0}$ & $Y_{0}$ & - \\
\hline
\end{tabular}

which relates the bubble radius oscillation with the internal pressure as $\Delta \widetilde{p}_{b}=-\Phi \Delta \widetilde{R}$ and $\widetilde{J}_{0}=J_{0} /\left(\rho_{b, 0} \omega R_{0}\right)$ is the total dimensionless mass-transfer flux accross the bubble interface such that $J(t)=J_{0} \Delta R \mathrm{e}^{i \omega t}$. The operators $\mathfrak{R}(\cdot)$ and $\mathfrak{J}(\cdot)$ indicate respectively the real and imaginary parts of the complex numbers.

The linearized analytical solution of the full model described in Section 2 yields the following expressions for the transfer function and the total, dimensionless mass-transfer flux:

$$
\Phi=\frac{3 \gamma}{1-3 i(\gamma-1) f_{\mathrm{Pe}}(1-\zeta)+3 \gamma i \widetilde{J}_{c}\left(\widetilde{H}_{v} \zeta-1\right)} ;
$$

with:

$$
\begin{gathered}
\zeta=\widetilde{\lambda} \frac{f_{\mathrm{Pe}}+\widetilde{J}_{c} \mathrm{Pe}_{b} \widetilde{H}_{v} \gamma(\gamma-1)^{-1}}{1+\sqrt{i \mathrm{Pe}_{l}}+\widetilde{\lambda}\left[f_{\mathrm{Pe}}+\widetilde{J}_{c} \mathrm{Pe}_{b} \widetilde{H}_{v}^{2} \gamma(\gamma-1)^{-1}\right]} ; \\
f_{\mathrm{Pe}}=\mathrm{Pe}_{b}^{-1}\left(\sqrt{i \mathrm{Pe}_{b}} \operatorname{coth}\left(\sqrt{i \mathrm{Pe}_{b}}\right)-1\right) .
\end{gathered}
$$

In these forms, $\mathrm{Pe}_{b}$ and $\mathrm{Pe}_{l}$ are the bubble and liquid Peclet numbers, $\widetilde{H}_{v}$ is the dimensionless enthalpy of evaporation/condensation and $\widetilde{\lambda}$ is the thermal conductivity ratio (see definitions in Tab. 1 ); $\zeta$ is a complex function that is proportional to the temperature variations at the bubble interface given by

$$
\widetilde{T}_{l}(\widetilde{r}=1)=1+\frac{\gamma-1}{\gamma} \zeta\left(\widetilde{p}_{b}(t)-1\right),
$$

and $\widetilde{J}_{c}$ is a characteristic mass flux across the bubble interface (the function $f_{\mathrm{Pe}}$ has been defined only for compactness).

In this work we focus on regimes where equilibrium conditions prevail at the interface at every instant, so that the kinetics of the phase change process is neglected and the characteristic mass flux used in Eq. 12 is [12]:

$$
\widetilde{J}_{c}=\frac{\operatorname{Sh}_{b}}{\sqrt{i \operatorname{Sh}_{b}} \operatorname{coth}\left(\sqrt{i \operatorname{Sh}_{b}}\right)-1} \frac{1-Y_{0}}{Y_{0}},
$$

which is a function of the bubble Sherwood number $\mathrm{Sh}_{b}$ (see definition in Table 1) and of the initial vapor content $Y_{0}$. This flux accounts only for the diffusion of the vapor through the interface, neglecting kinetic contributions due to the phase change process itself. This approximation holds when kinetic vaporization effects are much faster than the diffusive ones, such as for the size of the bubbles and the characteristic forcing frequencies considered here. It is important to remark that the total mass transfer flux $\widetilde{J}_{0}$ of Eq. (12) depends both on mass diffusion and thermal effects through its dependence on the dimensionless enthalpy of vaporization, the conductivity ratio appearing in $\zeta$ and the transfer function $\Phi$.

Numerically, it is possible to obtain the value of the transfer function running the simulation until steady state is reached. The number of oscillation cycles required to reach the steady state depend on the forcing frequency (bubble's Peclet number). The higher the Peclet number, the larger the number of cycles to reach steady state is. In the numerical simulations we obtained values ranging from 2 cycles for $\mathrm{Pe}_{b}=10^{-3}$ to $10^{4}$ cycles for $\mathrm{Pe}_{b}=10^{5}$ in the linear regime (where rectified diffusion effects can be disregarded as they arise in second order). Once the steady state is reached, the radius and pressure oscillation data is made dimensionless and fitted with a complex variable in the form $a \mathrm{e}^{i \omega t}$, which gives the solution in the frequency domain for the comparison. The convergence of the numerical solution with the number of nodes and the time step has been studied over the full range of the bubble Peclet number. The comparison between the analytical solution and the numerical values obtained for the real and imaginary parts of the transfer function $\Phi$ for an air- 


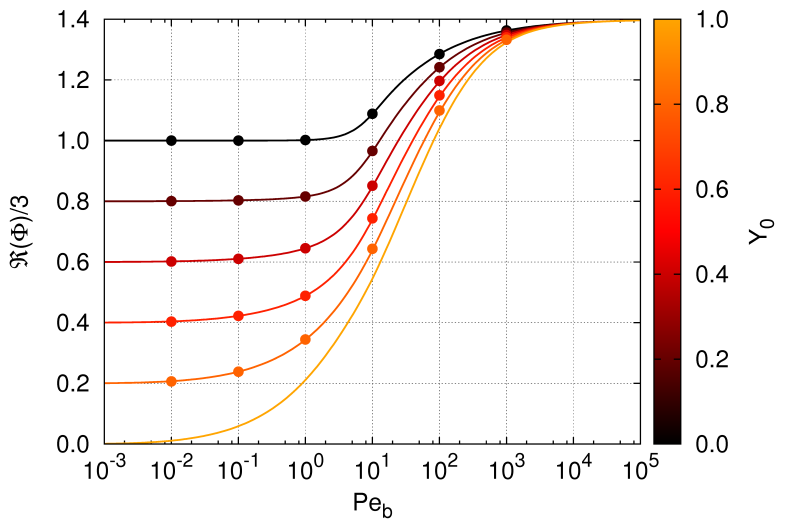

(a)

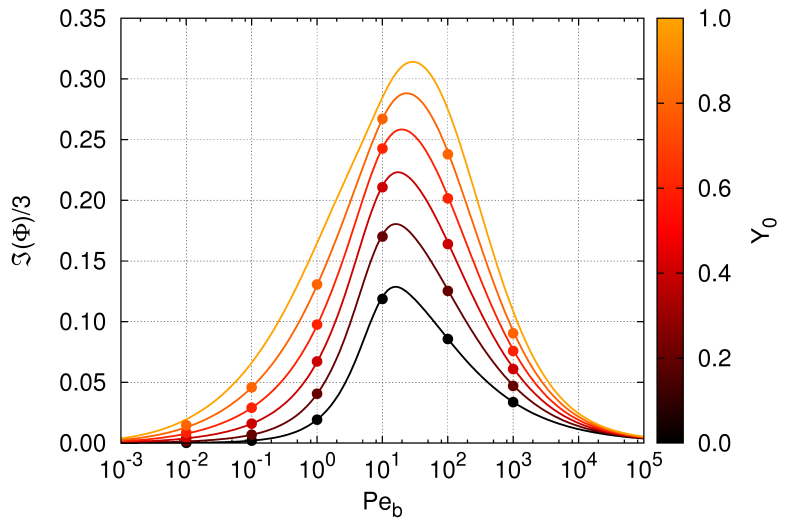

(b)

Figure 1: Validation of the numerical solution (points) against the analytical solution (lines) of the transfer function $\Phi$ for the full model: (a) real and (b) imaginary part.

water system is shown in Fig. 1. The real part of this function is of particular concern, as it can be physically interpreted as an effective polytropic index, which, for air-water systems, varies between 1 and $\gamma_{g}$ for pure gas bubbles and between 0 and $\gamma_{v}$ for pure vapor bubbles (in the isothermal and adiabatic limits respectively). We obtain excellent agreement of the numerical data with the analytical solution in the full parameter range tested in this work. The dimensionless flux across the interface numerically obtained is also compared in Figure 2 showing excellent agreement with the theoretical predictions.

\section{LINEAR OSCILLATION REGIMES}

In this section we discriminate between the various physical effects influencing the response of the bubble and the mass transfer flux by comparing the solution obtained with the full model with those obtained with various simplified models.

\subsection{The full-equilibrium model: slow bubble oscilla- tions}

The range of applicability of the full-equilibrium model is interesting given its widespread utilization in various situations involving phase change. This model is obtained assuming that: (a) the characteristic oscillation velocity is smaller than the characteristic heatdiffusion velocity inside the bubble $\left(\mathrm{Pe}_{b} \ll 1\right)$, which guarantees uniform temperature inside the bubble; (b) the characteristic oscillation velocity is smaller than the characteristic mass diffusion velocity inside the bubble $\left(\mathrm{Sh}_{b} \ll 1\right)$, which guarantees that the vapor concentration inside the bubble is uniform; (c) that the interface's temperature is constant, that is, the characteristic velocity of phase change is sufficiently slow to avoid any significant change on the temperature of the interface. This last condition imposes that $\mathrm{Pe}_{b}$ has to be smaller than a critical Peclet number $\mathrm{Pe}_{b}^{c r}$, below which temperature variations at the interface are negligible. The exact expression for the critical Peclet number is derived in [12] and is shown to depend on the ratio between the bubble/liquid thermal conductivities and on the enthalpy of vaporization:

$$
\mathrm{Pe}_{b}^{c r}=3 \frac{\widetilde{\lambda}}{\widetilde{H}_{v}^{2}} \frac{1-Y_{0}}{Y_{0}} \frac{\gamma-1}{\gamma} .
$$

Under these conditions, the bubble's pressure is given by

$$
p_{b}=p_{g, 0}\left(\frac{R_{0}}{R}\right)^{3}+p_{v}\left(T_{\infty}\right),
$$

and the transfer function becomes $\Phi(\omega \rightarrow 0)=3(1-$ $\left.Y_{0}\right)$. The general expression for the total mass transfer flux across the interface (Eq. 12) is simplified using the limiting expression of Eq. 16 for $\mathrm{Sh}_{b} \ll 1$ and $\zeta \approx 0$, so that $\widetilde{J}_{c} \approx \frac{1}{3} i \frac{Y_{0}}{1-Y_{0}}$. In these circumstances, Eq. 12 simplifies to

$$
\widetilde{J_{0}}=Y_{0} i
$$

Figure 3 represents the isolines of the relative error of the modulus of the transfer function for the full- 


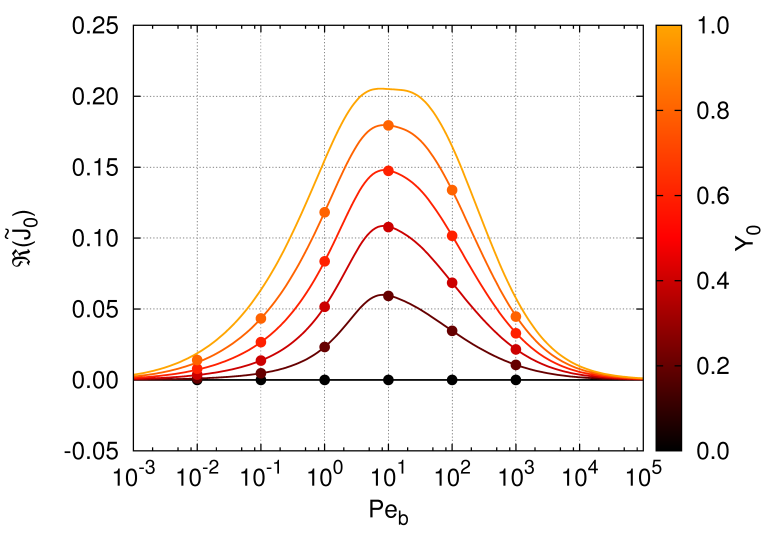

(a)

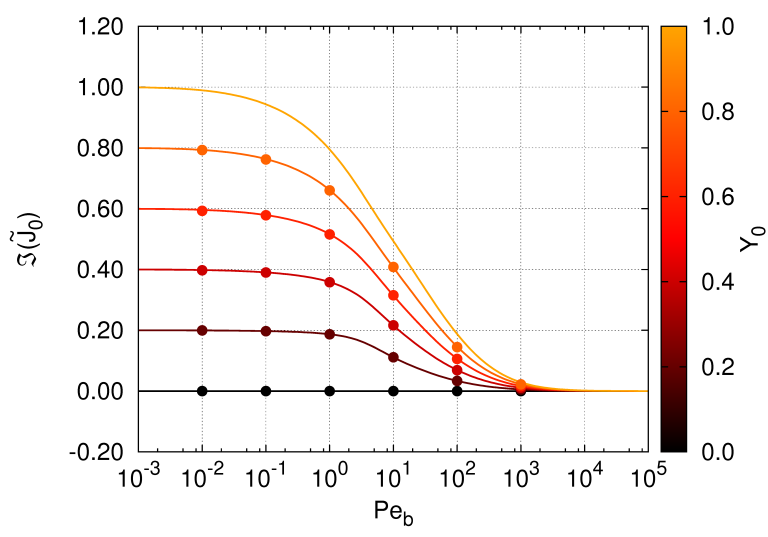

(b)

Figure 2: Validation of the numerical solution (points) against the analytical solution (lines) of the dimensionless total flux across the interface for the full model: (a) real and (b) imaginary part.

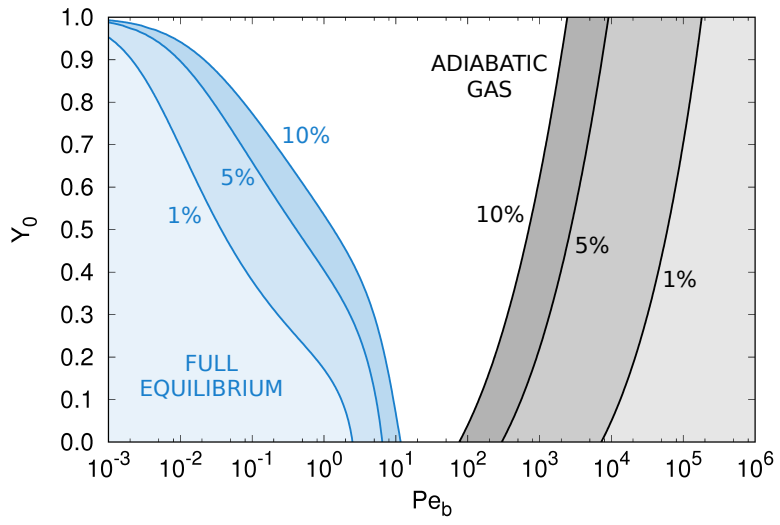

Figure 3: Isolines of the relative error of the approximations of the transfer function provided by the adiabatic model (in grey) and the fullequilibrium model (in blue). 
equilibrium model compared with the full expression,

$$
\epsilon=\frac{\left|\Phi(\omega \rightarrow 0)-\Phi_{\text {full }}\right|}{\Phi_{\text {full }}}
$$

where $\Phi_{\text {full }}$ corresponds to the expression of Eq. 11 .

As expected, the diagram shows that the fullequilibrium model correctly captures the bubble radial evolution with an error below $10 \%$ with respect to the full model in the low-frequency range. We also emphasize that as predicted by Eq. 17, the characteristic Peclet below which the full-equilibrium model is applicable is smaller when the vapor content is large.

\subsection{The adiabatic model: fast bubble oscillations}

In the adiabatic gas model neither heat or mass are exchanged across the interface and the equation of state relating bubble's pressure and volume changes is

$$
p_{b}=p_{b, 0}\left(\frac{R_{0}}{R}\right)^{3 \gamma}
$$

and thefore, $\Phi_{\mathrm{ad}}=3 \gamma$. Figure 3 represents the range of validity of this approximation using the iso-error lines obtained by comparing $\Phi_{\text {ad }}$ with the full-model expresion given in Eq. 11. As expected, at high frequencies the adiabatic model provides a good representation of the bubble response as heat- and mass-transfer effects across the interface can be neglected in order to correctly predict the bubble's pressure evolution. In this regime, the water vapor is trapped inside the bubble given that it has no time to diffuse. This effect, identified in simulations of strong non-linear collapses [29, 30], has been found to have important implications on the sonochemical activity [31,32], being an important ingredient in models able to reproduce experimental data for violent collapse of gas-vapor bubbles [33]. The amount of vapor trapped in the linear regime is quantified here defining a vapor trapping parameter $\eta_{\text {trap }}$ as the relative difference between the total mass transfer flux across the bubble interface, $\widetilde{J}_{0}$, and the mass transfer flux across the interface assuming equilibrium conditions (Eq. 19),

$$
\eta_{\text {trap }}=\frac{\left|\widetilde{J}_{0}-\widetilde{J}_{0, e q}\right|}{\left|\widetilde{J}_{0, e q}\right|} .
$$

Figure 4 shows this parameter as a function of the bubble Sherwood number and of the vapor content. As expected, the amount of vapor trapped increases at large frequencies (high Sherwood numbers). Remarkably, the dimensionless amount of the trapped vapor only depends slightly on the vapor content for Sherwood numbers between $10^{-2}$ to $10^{2}$.

\subsection{Liquid-heat diffusion regime}

It is well known that the heat diffusion in the liquid surrounding the bubble can control the total flux across the interface and therefore the dynamic response of the bubble. These effects can be captured using the full expression for $\zeta$ still imposing the limiting expression of $\widetilde{J}_{c} \approx \frac{1}{3} i \frac{Y_{0}}{1-Y_{0}}$ in order to neglect any effect of vapor diffusion on the solution. Thus we find

$$
\begin{gathered}
\Phi_{\mathrm{LHD}}=\frac{3 \gamma}{1-3 i(\gamma-1) f_{\mathrm{Pe}}(1-\zeta)-\gamma \frac{Y_{0}}{1-Y_{0}}\left(\widetilde{H}_{\nu} \zeta-1\right)} \\
\widetilde{J}_{0, \mathrm{LHD}}=\frac{1}{3} i \frac{Y_{0}}{1-Y_{0}}\left(1-\widetilde{H}_{\nu} \zeta\right) \Phi_{\mathrm{LHD}}
\end{gathered}
$$

where the subindex LHD denotes liquid-heat diffusion model.

Figure 5 depicts the iso-error lines of the approximated expression for the modulus of the transfer function compared to the exact expression (Eq. 11). As we can see this model works remakably well for a wide range of situations in the Peclet-number/vapor-content plane. The model includes the full equilibrium model and the adiabatic model as particular solutions for the case of a pure gas bubble. Note that although the limiting expression used for the mass transfer flux assumes that $\mathrm{Sh}_{b} \ll 1$, the impact of the flux on the dynamic response of the system at high frequencies is not relevant as the characteristic dimensionless mass-transfer velocity is small compared to the expansion velocity, $\widetilde{J_{0}} \ll 1$ (see Figure 2 ). In other words, the dynamics of the bubble at high frequencies is controlled by the expansion/compression of the bubble rather than the phase change process. Only at intermediate frequencies and conditions where the content of water vapor and immiscible gas are similar, the model is not able to correctly capture the response of the bubble. As we will show next, in these conditions the vapor diffusion plays an important role on the dynamic response of the bubbles.

The dimensionless interface's temperature variations $\zeta$ and the thickness of the thermal boundary layer in the liquid $\delta_{l}$ are relevant parameters when liquid's heat diffusion controls the response of the bubble. We can ob- 


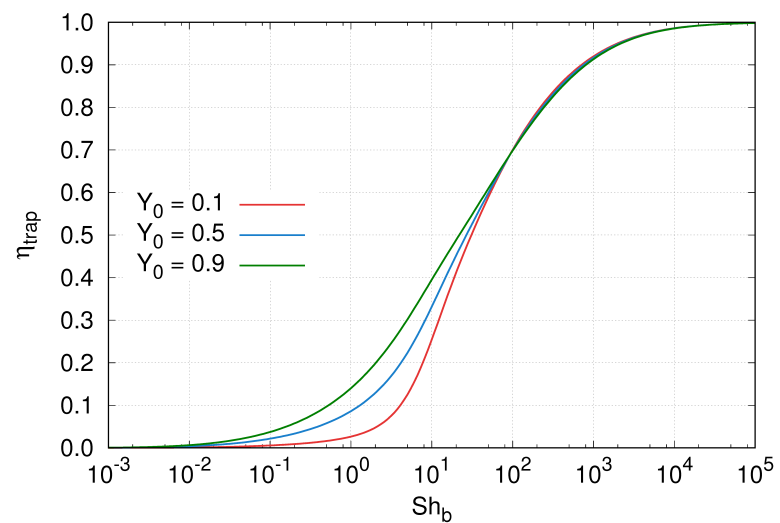

Figure 4: Measurement of the trapped vapor content as a function of the bubble Sherwood number for $Y_{0}=0.1,0.5,0.9$. The bubble radius is $R_{0}=10 \mu \mathrm{m}$

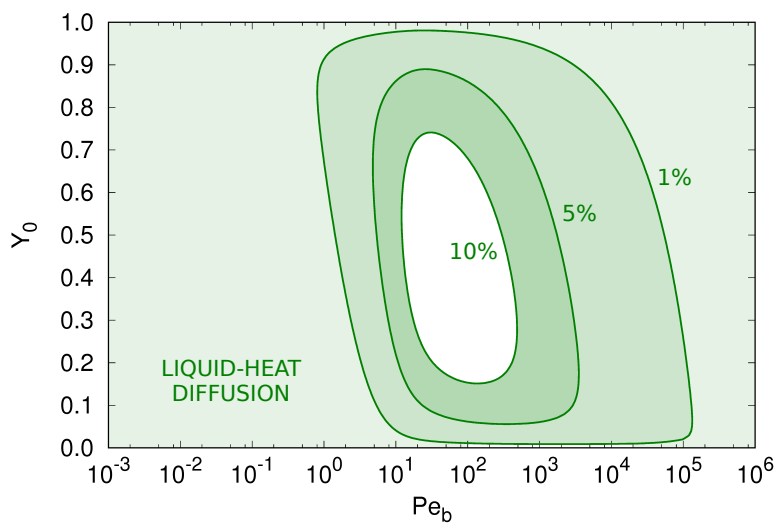

Figure 5: Range of applicability of the liquid-heat diffusion model. The lines represent iso-error lines obtained by the comparison of the simplified and full expressions of the modulus of the transfer function (Eqs. 23 and 11 respectively). 
tain the fluctuation of the liquid's temperature with respect to the interface's temperature variation as

$$
\frac{\widetilde{T}_{l}(\widetilde{r})-1}{\left.\widetilde{T}_{l} \widetilde{r}=1\right)-1}=\frac{e^{\sqrt{i \mathrm{Pe}_{l}(1-\widetilde{r})}}}{\widetilde{r}} .
$$

Figure 6 shows the dependence of the thickness of the thermal boundary layer in the liquid, taken as the distance at which $\widetilde{T}_{l}\left(\delta_{l} / R_{0}\right)-1=0.01\left(\widetilde{T}_{l}(\widetilde{r}=1)-1\right)$. For completeness, this figure also includes the dimensionless temperature variation $\zeta$ as a function of the Peclet's number. The boundary layer thickness takes its largest values at low frequencies, reaching an asymptotic value of 100 times the bubble radius for Peclet's number below $10^{-8}$. This parameter does not depend on the amount of vapor because of the normalization chosen in Eq. (25). The influence of the vapor content can be clearly seen in the parameter $\zeta$. The larger interface's temperature fluctuations are found for pure vapor bubbles in the low frequency limit which can be analytically shown to be $\zeta\left(\mathrm{Pe}_{b} \rightarrow 0\right)=\widetilde{H}_{v}^{-1}$. This value corresponds to the limiting case where the interface follows the saturation curve. As the relative amount of vapor decreases, the interface's temperature fluctuations are significantly attenuated. In addition, for a constant gas/vapor content we observe the appearance of a peak value for intermediate values of the Peclet number. This peak shifts towards larger frequencies when decreasing the vapor content.

\subsection{Vapor-mass diffusion regime}

In order to complete the analysis of the range of validity of simplified approaches we consider here the vapor mass diffusion regime proposed by Preston et al [34] where the total mass transfer flux across the interface is given by the diffusion of the vapor inside the bubble. This model neglects any influence of heat diffusion in the thermal boundary layer surrounding the bubble and therefore, it assumes the interface to be at constant temperature $(\zeta \approx 0)$. In this case, the simplified expressions for the transfer function and the total flux are

$$
\begin{gathered}
\Phi_{\mathrm{MD}}=\frac{3 \gamma}{1-3 i(\gamma-1) f_{\mathrm{Pe}}(1-\zeta)-3 \gamma i \widetilde{J}_{c}} ; \\
\widetilde{J}_{0, \mathrm{MD}}=\widetilde{J}_{c} \Phi_{\mathrm{MD}}
\end{gathered}
$$

The range of applicability of this approach in the Peclet-number/vapor-content plane is represented in Figure 7. As we can see this model also includes the full-equilibrium model and the adiabatic model as particular solutions for pure gas bubbles. For sufficiently small vapor contents, the model predicts well the transition between the two models for the full range of frequencies. Only for large vapor contents, liquid-heat diffusion takes control of the flux and this model is unable to correctly predict the bubble radius evolution. Within the range of applicability of this regime, the gas exerts a strong resistance to the process of vapor diffusion and controls the total mass flux irrespective of the heat transport process in the liquid boundary layer. These observations become clear in Fig. 8, where we compare the predictions of the simplified models with the exact solution for $\Phi$. The high frequency limit corresponds to the limit of an adiabatic transformation, while the low frequency limit corresponds to the isothermal limit of a liquid/vapor mixture where the transfer function becomes $\Phi(\omega \rightarrow 0)=3\left(1-Y_{0}\right)$. While the liquid-heat diffusion model predicts better the transition between the isothermal and adiabatic limit for large vapor contents $\left(Y_{0}=0.8\right)$ the vapor-mass diffusion model performs better for small vapor concentrations $\left(Y_{0}=0.2\right)$. It also becomes clear that the Peclet number required to reach the low-frequency limit (where all the models converge to the full-equilibrium model) decreases when increasing the vapor content from 0.2 to 0.8 . This is consistent with the fact that the critical Peclet number of Eq. (17) is 16 times smaller in the case of $Y_{0}=0.8$ than in the case of $Y_{0}=0.2$.

\subsection{Summary of linear oscillation regimes}

Using the simplified models described above we propose in Figure 9 full oscillation diagrams for a 10 $\mu \mathrm{m}$ and $1 \mathrm{~mm}$ bubble taking the isolines for $10 \%$ error. In the case of a coexistence of models we priviledge the simplest one. As previously discussed, the results are especially interesting in the intermediate-to-low frequency range, when transient effects related to heat and mass diffusion arise and play an important role on the bubble oscillation. On the one hand, for high vapor contents, the bubble oscillation is controlled by heat-diffusion effects in the liquid, that is, the mass flux across the interface is limited by the energy required to vaporize the liquid, which is controlled in turn by the heat transport in the liquid boundary layer. On the other hand, for low vapor contents, there exists a regime where the dynamic response of the bubble is controlled by vapor-mass diffusion effects inside the bubble (red region) rather than by heat diffusion effects in the liquid. Note that we also identify regions where a concurrency between heat and mass diffusion occurs and no approximation applies (FULL model region). At high frequencies, $\mathrm{Pe}_{b}>10$, the dynamic response of 


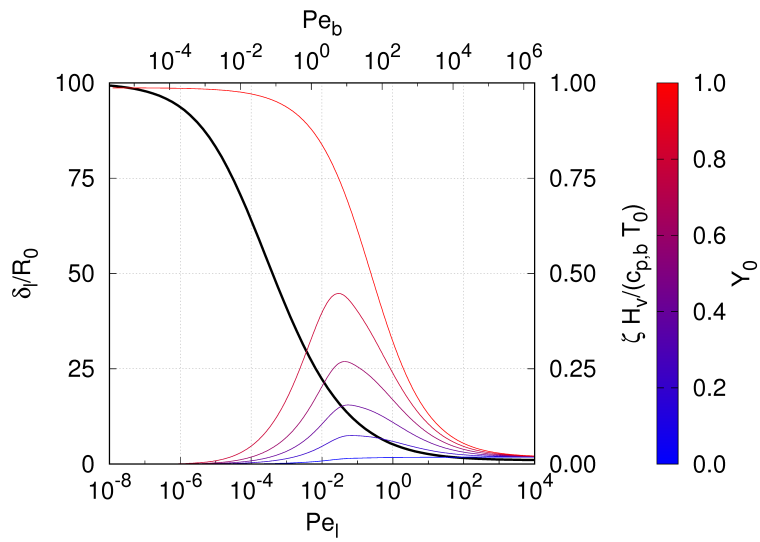

Figure 6: Black line: Thickness of the thermal boundary layer in the liquid $\delta_{l} / R_{0}$ as a function of the Peclet's number. Color lines: Dimensionless interface's temperature fluctuations $\zeta H_{v}^{*}$ as a function of the liquid's Peclet number and vapor content.

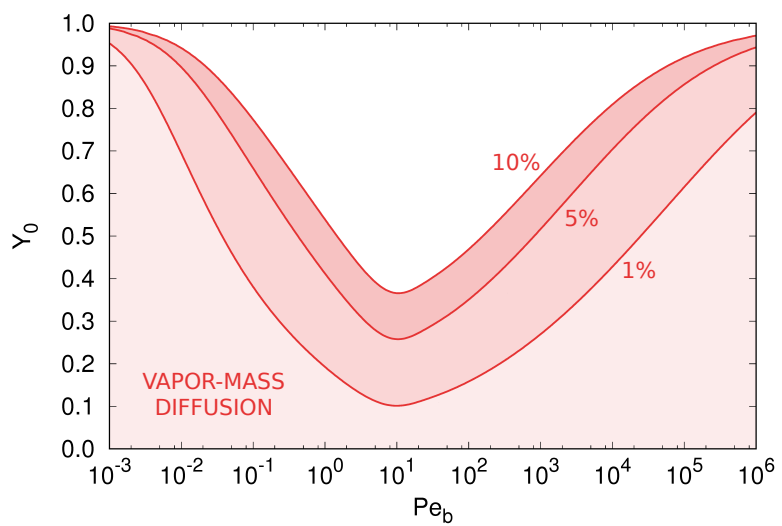

Figure 7: Range of applicability of the vapor-mass diffusion model. The lines represent iso-error lines obtained by the comparison of the simplified (Eq. 26) and full expression (Eq. 11) of the modulus of the transfer function.

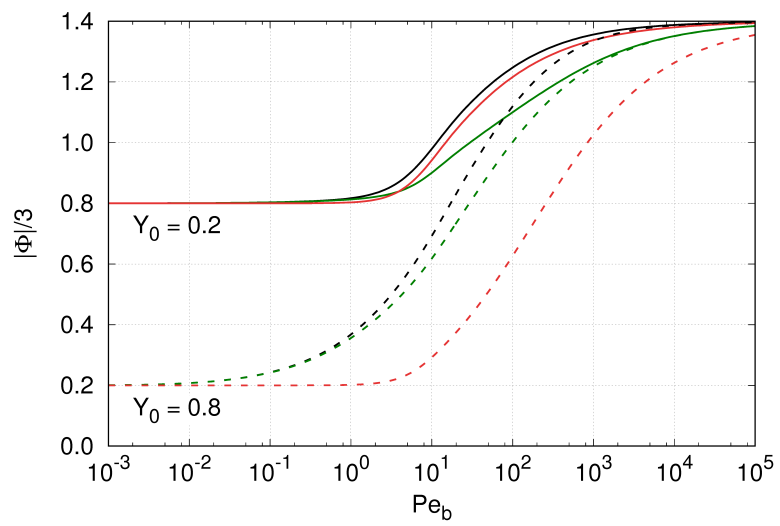

Figure 8: Modulus of the transfer function obtained with the full model (black), the liquid-heat diffusion model (green) and the mass-diffusion model (red) for vapor content $Y_{0}=0.2$ (solid lines) and $Y_{0}=0.8$ (dashed lines). 


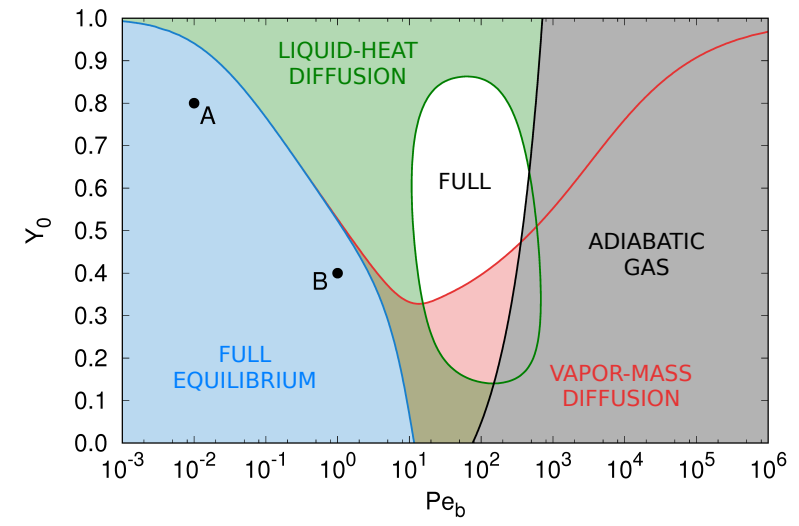

(a)

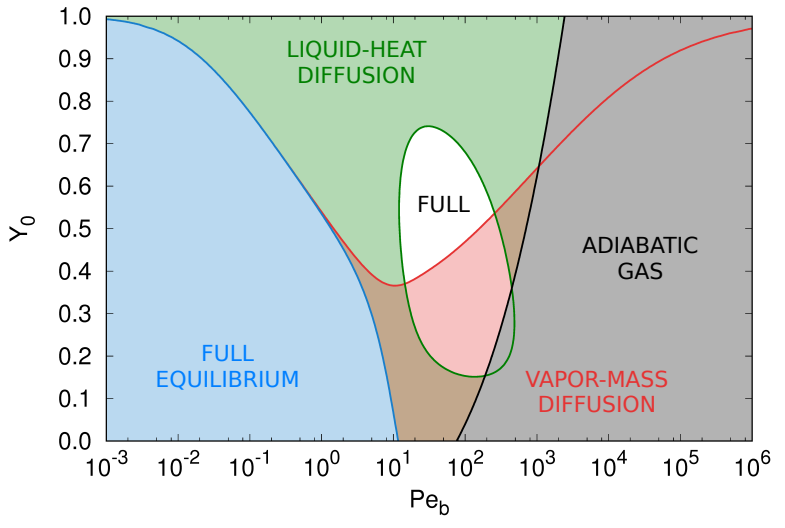

(b)

Figure 9: Linear oscillation regimes according to the error (10\% with respect to the full model) on the magnitude of the transfer function $|\Phi|$ for two different bubble radii: (a) $R_{0}=10 \mu \mathrm{m}$ and (b) $R_{0}=1 \mathrm{~mm}$. Numerical simulations for the time evolution of the bubble radius at points A and $\mathrm{B}$ in (a) are shown in Fig. 10 and 11.

the bubble is no longer influenced by mass transfer and the adiabatic model applies.

\section{LINEAR TO NON-LINEAR TRANSITION}

Based on the theoretical diagrams obtained for the linear regime and on the validation of the numerical code presented in Section 3.2, in this section we investigate the influence of the vapor content on the non-linear response of bubbles. To this purpose, we show and discuss the evolution of the bubble radius obtained numerically and the local orbits described by the volumeaveraged vapor content inside the bubble $\bar{Y}$ and an instantaneous Peclet number. This latter quantity is defined as the ratio between the oscillation velocity and the characteristic heat transfer rate $\Gamma_{t, b} / R(t)$ :

$$
\mathrm{Pe}_{b, i}:=\frac{3}{4 \pi} \frac{c_{p, b}}{\lambda_{b}} \frac{\dot{R}(t)}{R^{2}(t)} m(t)
$$

being $m(t)$ the instantaneous (total) mass of the bubble. For reference, we will include in the analysis the solution provided by the full-equilibrium model given that it is widely-used and all transient effects related to heat diffusion in the surrounding liquid and the vapor-mass diffusion inside the bubble are neglected.

In Fig. $10 \mathrm{a}$ and $10 \mathrm{~b}$ we show the results obtained for $\mathrm{Pe}_{b}=0.01, Y_{0}=0.8, R_{0}=10 \mu \mathrm{m}$, corresponding to point $\mathrm{A}$ in the diagram of Fig. 9a. For reference, we show the critical vapor fraction, $Y_{c r}$, defined as the value beyond which, for a given Peclet number, the error is equal to $10 \%$ in the linear regime (obtained from Fig. 9a). The dimensionless pressure amplitudes $\Delta \widetilde{p}_{\infty}$ tested range from 0.1 to 0.16 . In these conditions, while pressure amplitudes are well below previously reported values for non-linear oscillation of pure gas bubbles [35], non-linear effects become apparent while the oscillation is not chaotic yet. When the perturbation is smaller than a critical threshold (e.g. $\Delta \widetilde{p}_{\infty}=0.10$ ) the local orbit is completely within the full-equilibrium regime in the phase diagram and the full-equilibrium model provides a good approximation of the exact solution. For $\Delta \widetilde{p}_{\infty}=0.13$, the orbit gets closer to the critical vapor content, transient heat-diffusion effects in the liquid become non-negligible and the error becomes important. Finally, the error blows up for $\Delta \widetilde{p}_{\infty}=0.16$, where the vapor content oscillates outside the regime of validity of the full-equilibrium model and the solution provided by this model diverges from that obtained from the full model. Figure $10 \mathrm{~b}$ reveals that transient diffusion in the liquid boundary layer around the bubble has a strong damping influence on the bubble response when increasing the pressure amplitude, which stresses the importance of modeling the liquid-heat diffusion in situations where the vapor content reaches large values.

A similar response is observed in Fig. 11a and $11 \mathrm{~b}$ for $\mathrm{Pe}_{b}=1, Y_{0}=0.4$ and $R_{0}=10 \mu \mathrm{m}$, corresponding to point $\mathrm{B}$ in the diagram of Fig. 9a. If the amplitude of the external pressure wave is such that the orbit is fully within the range of validity of the 


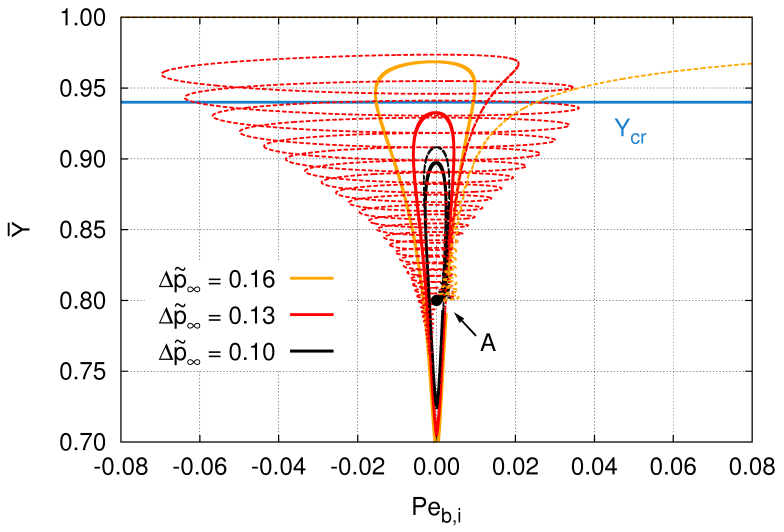

(a)

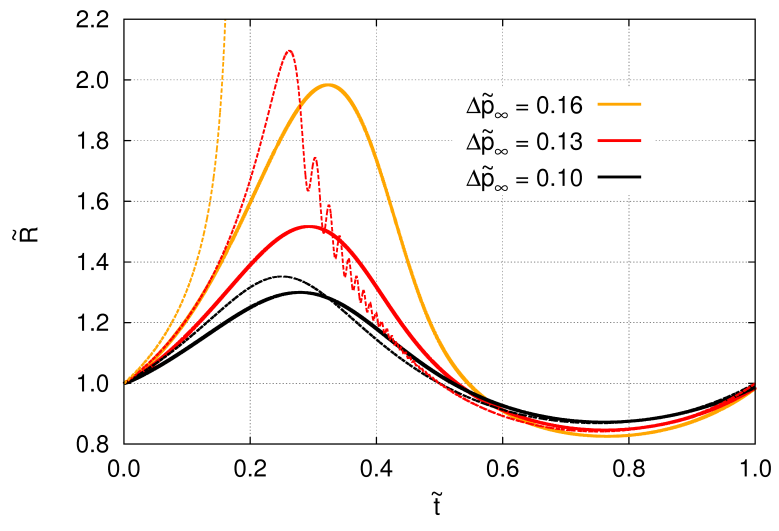

(b)

Figure 10: Analysis of the bubble response to external pressure pulses for the point A in the diagram of Fig. 9a. (a) Orbits described by the average vapor content and the instantaneous Peclet number. For reference, the critical vapor fraction given by the boundary between the full-equilibrium and the liquid-heat diffusion model in Fig. 9a is included; (b) dimensionless radius $\widetilde{R}=R / R_{0}$ as a function of the dimensionless time $\widetilde{t}=t \omega /(2 \pi)$ (first oscillation period). Solid and dashed lines represent respectively the solution given by the full model and the full-equilibrium model.

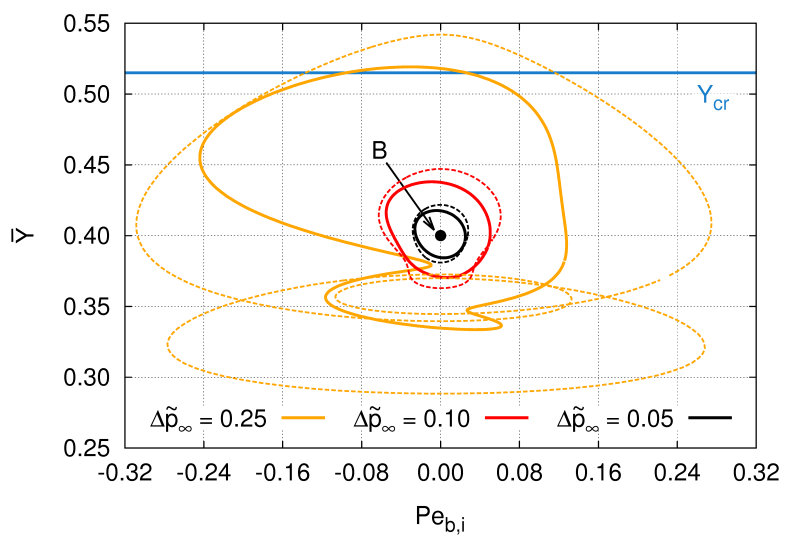

(a)

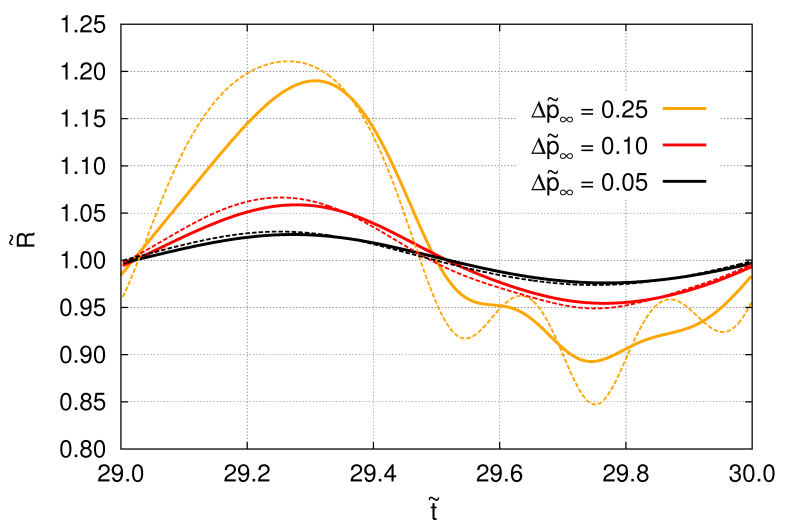

(b)

Figure 11: Analysis of the bubble response to external pressure pulses for the point B in the diagram of Fig. 9a. (a) Orbits described by the average vapor content and the instantaneous Peclet number. For reference, the critical vapor fraction given by the boundary between the full-equilibrium and the liquid-heat diffusion model in Fig. 9a is included; (b) dimensionless radius as a function of the dimensionless time (30-th oscillation period). Solid and dashed lines represent respectively the solution given by the full model and the full-equilibrium model. 
full-equilibrium model the dynamic response of the bubble is well predicted. As soon as the critical pressure amplitude is exceeded, the errors on the oscillation orbits become significant and unphysical oscillations are predicted by the full-equilibrium model.

In order to provide a general overview about the importance of transient heat- and mass-transfer effects on the (weakly) non-linear response of the bubble, we represent the difference between the full-equilibrium and the full model by the L1-norm error:

$$
\|\widetilde{R}\|_{1}=\frac{1}{t_{e}} \int \frac{\left|R_{F E}-R_{F}\right|}{R_{0}} d t,
$$

where $t_{e}$ is the final simulation time, $R_{F E}$ and $R_{F}$ are the radius given by the full-equilibrium model and the full model respectively.

As shown in Fig. 12a, for high Peclet numbers $\left(\mathrm{Pe}_{b}=100\right)$ the error does not depend significantly on the vapor content because the mass transfer flux does not have a strong influence neither on the bubble interface evolution nor in the change of the bubble's mass. At low Peclet numbers, mass transfer effects become relevant and the bubble dynamic evolution strongly depends on the vapor content. Fig. 12b shows the evolution of the dimensionless quantity $\|\widetilde{R}\|_{1} / \Delta \widetilde{p}$ as a function of the amplitude of the pressure disturbance and of the vapor content for a low Peclet number $\left(\mathrm{Pe}_{b}=0.01\right)$. We can see that the dimensionless error given by the full-equilibrium model reaches an asymptotic value for low-amplitude disturbances. This asymptote depends on the vapor content and on the bubble Peclet number. This analysis shows that the dynamic response for pure gas bubbles is well reproduced by an isothermal model even for $\Delta \widetilde{p} \approx 1$, while for bubbles containing a large amount of vapor it becomes critical to model transient heat-transport effects in the surrounding liquid when $\Delta \widetilde{p}$ is below a certain critical dimensionless pressure amplitude $\Delta \widetilde{p}_{\infty}^{c r}$. The critical amplitude below which the error reaches an asymptotic value is numerically obtained and shown in Fig. 12b. As clearly seen, it becomes smaller as the vapor content is increased. For instance, for the largest vapor fraction tested here, $Y_{0}=0.8$, the critical dimensionless pressure amplitude beyond which the error blows up is 0.1 .

\section{CONCLUSIONS}

In this work we have presented an analysis of heatand mass-diffusion effects on the oscillating dynamics of gas-vapor bubbles. Based on analytical solutions, we have proposed phase diagrams for the oscillation regimes of air/water systems in the linear limit. Using the diagrams we have shown that, even at very low frequencies, transient heat diffusion in the liquid and mass diffusion inside the bubble can play an important role on the dynamic response of the bubble. The accuracy of the proposed regimes has been assessed beyond the linear limit by means of numerical solutions. The code, validated against the theoretical solutions in the linear limit, has been used to obtain the orbits described by the instantaneous bubble properties. We have discussed the importance of accurate modeling of transient effects in various regimes predicted by the linear theory and shown that non-linearity severely restricts the validity of the full-equilibrium assumption. In particular we have observed that, as soon as the bubble is filled with vapor, it becomes critical to model transient heat-diffusion effects in the bubble surroundings, which act as a strong damping mechanism.

This work is meant to clarify in a systematic way the range of applicability of the most commonly-adopted models in bubble dynamics. Given the relevance of the dynamics of gas-vapor bubbles in cavitating flows, we expect this work to motivate further efforts in the development of simplified and/or reduced-order models (such as those proposed by Preston et al [34]) accounting for the influence of liquid-heat diffusion on the dynamic response of the bubbles.

\section{Acknoweledgements}

This work has been financially supported by the PERSU program (Sorbonne Universités) and the ANR CachMap project (ANR-15-ASTR-0026-02).

[1] E. Brujan, Cavitation in Non-Newtonian Fluids: With Biomedical and Bioengineering Applications, 2011th Edition, Springer, 2010.

[2] W. Lauterborn, T. Kurz, R. Mettin, C. D. Ohl, Experimental and theoretical bubble dynamics, Adv. Chem. Phys. 110 (1999) 295380 .

[3] K. W. Commander, A. Prosperetti, Linear pressure waves in bubbly liquids: Comparison between theory and experiments, J. Acoust. Soc. Am. 85 (1989) 732.

[4] C. E. Brennen, Cavitation and bubble dynamics, Cambridge University Press, 2013.

[5] C. Coste, C. Laroche, S. Fauve, Sound propagation in a liquid with vapour bubbles, EPL (Europhys. Lett.) 11 (4) (1990) 343347. 


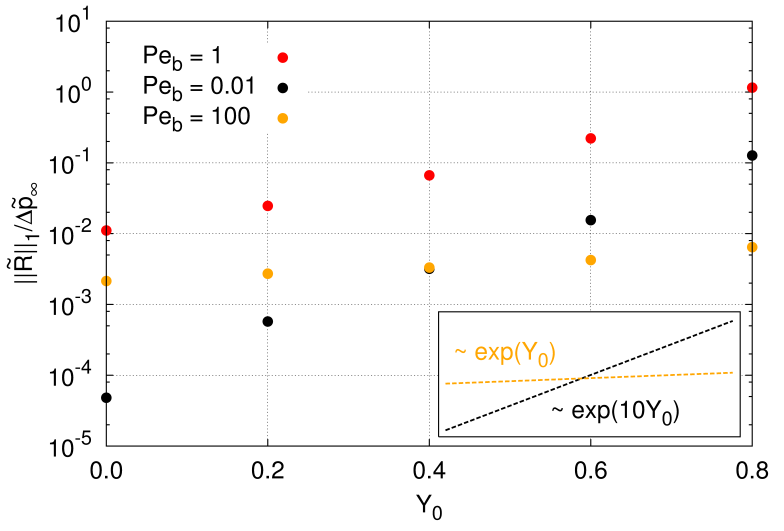

(a)

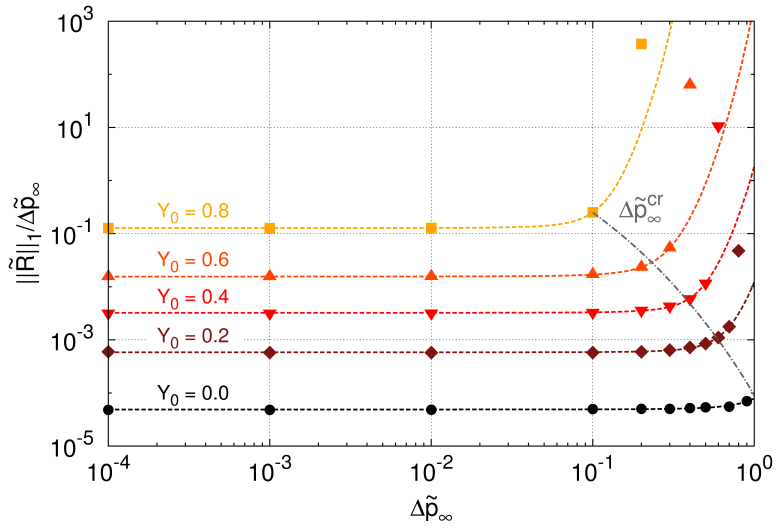

(b)

Figure 12: Analysis of the L1-norm error of Eq. (29) given by the full-equilibrium with respect to the full model: (a) as a function of the vapor content and of the Peclet number in the low-amplitude oscillation limit $\left(\Delta \widetilde{p}_{\infty}=10^{-3}\right)$; (b) as a function of the pressure amplitude and vapor content for $\mathrm{Pe}_{b}=0.01$ (linear to non-linear transition). The grey dashed line represents the critical pressure amplitude, numerically fitted, beyond which the error of the full-equilibrium model blows up.

[6] C. E. Brennen, Fundamentals of Multiphase Flows, 1st Edition, Cambridge University Press, 2009.

[7] R. Saurel, O. Lemetayer, A multiphase model for compressible flows with interfaces, shocks, detonation waves and cavitation, J. Fluid Mech. 431 (2001) 239-271.

[8] H. Shamsborhan, O. Coutier-Delgosha, G. Caignaert, F. Abdel Nour, Experimental determination of the speed of sound in cavitating flows, Exp. Fluids 49 (6) (2010) 1359-1373.

[9] C. Brennen, The dynamic behavior and compliance of a stream of cavitating bubbles, J. Fluids Eng. 95 (4) (1973) 533-541.

[10] S. Watanabe, T. Hidaka, H. Horiguchi, A. Furukawa, Y. Tsujimoto, Steady analysis of the thermodynamic effect of partial cavitation using the singularity method, J. Fluids Eng. 129 (2) (2007) 121-127.

[11] A. Prosperetti, The speed of sound in a gas-vapour bubbly liquid, Interface Focus 5 (5) (2015) 20150024.

[12] D. Fuster, F. Montel, Mass transfer effects on linear wave propagation in diluted bubbly liquids, J. Fluid Mech. 779 (2015) 598621.

[13] A. Prosperetti, Vapor bubbles, Annu. Rev. Fluid Mech. 49 (2017) 221-248.

[14] M. A. Ainslie, T. G. Leighton, Review of scattering and extinction cross-sections, damping factors, and resonance frequencies of a spherical gas bubble, J. Acoust. Soc. Am. 130 (5) (2011) 3184-3208.

[15] D. Legendre, J. Borée, J. Magnaudet, Thermal and dynamic evolution of a spherical bubble moving steadily in a superheated or subcooled liquid, Phys. Fluids (1994-present) 10 (6) (1998) $1256-1272$.

[16] L. E. Scriven, On the dynamics of phase growth, Chem. Eng. Sci. 10 (1-2) (1959) 1-13.

[17] E. Ruckenstein, E. J. Davis, The effects of bubble translation on vapor bubble growth in a superheated liquid, Int. J. Heat Mass Transfer 14 (7) (1971) 939-952.

[18] N. A. Gumerov, Dynamics of vapor bubbles with nonequilibrium phase transitions in isotropic acoustic fields, Phys. Fluids 12 (1) (2000) 71-88.

[19] Y. Hao, A. Prosperetti, The dynamics of vapor bubbles in acous- tic pressure fields, Phys. Fluids 11 (8) (1999) 2008-2019.

[20] R. I. Nigmatulin, N. S. Khabeev, F. B. Nagiev, Dynamics, heat and mass transfer of vapour-gas bubbles in a liquid, Int. J. Heat Mass Transfer 24 (6) (1981) 1033 - 1044.

[21] D. Fuster, S. Zaleski, The importance of liquid evaporation on rectified diffusion processes, Proceedings of the 7th International Conference on Multiphase Flow (2010)

[22] N. A. Gumerov, Weakly linear oscillations of the radius of a vapour bubble in an acoustic field, J. Appl. Math. Mech. 55 (2) (1991) $205-211$.

[23] G. Hauke, D. Fuster, C. Dopazo, Dynamics of a single cavitating and reacting bubble, Phys. Rev. E 75 (2007) 066310.

[24] A. Prosperetti, A generalization of the Rayleigh-Plesset equation of bubble dynamics, Phys. Fluids 25 (3) (1982) 409-410.

[25] D.-Y. Hsieh, M. S. Plesset, Theory of rectified diffusion of mass into gas bubbles, J. Acoust. Soc. Am. 33 (2) (1961) 206-215.

[26] A. Eller, H. G. Flynn, Rectified diffusion during nonlinear pulsations of cavitation bubbles, J. Acoust. Soc. Am. 37 (3) (1965) 493-503.

[27] L. A. Crum, G. M. Hansen, Generalized equations for rectified diffusion, J. Acoust. Soc. Am. 72 (5) (1982) 1586-1592.

[28] D. Fuster, G. Hauke, C. Dopazo, Influence of accommodation coefficient on nonlinear bubble oscillations, J. Acoust. Soc. Am. 128 (2010) 5-10.

[29] B. D. Storey, A. J. Szeri, Water vapour, sonoluminescence and sonochemistry, Proceedings of the Royal Society of London A: Mathematical, Physical and Engineering Sciences 456 (1999) (2000) 1685-1709.

[30] S. Fujikawa, T. Akamatsu, Effects of the non-equilibrium condensation of vapour on the pressure wave produced by the collapse of a bubble in a liquid, J. Fluid Mech. 97 (1980) 481-512.

[31] W. C. Moss, D. A. Young, J. A. Harte, J. L. Levatin, B. F. Rozsnyai, G. B. Zimmerman, I. H. Zimmerman, Computed optical emissions from a sonoluminescing bubble, Phys. Rev. E 59 (1999) 2986-2992.

[32] W. C. Moss, D. B. Clarke, D. A. Young, Calculated pulse widths and spectra of a single sonoluminescing bubble, Science 276 (5317) (1997) 1398-1401. 
[33] T. J. Matula, P. R. Hilmo, B. D. Storey, A. J. Szeri, Radial response of individual bubbles subjected to shock wave lithotripsy pulses in vitro, Phys. Fluids 14 (3) (2002) 913-921.

[34] A. T. Preston, T. Colonius, C. E. Brennen, A reduced-order model of diffusive effects on the dynamics of bubbles, Phys. Fluids 19 (12) (2007) 3302.

[35] W. Lauterborn, T. Kurz, Physics of bubble oscillations, Rep. Prog. Phys. 73 (10) (2010) 106501. 